Part of Journal of Research of the National Bureau of Standards, Volume 23, August 1939

\title{
ELECTROPHORESIS OF COLLAGEN
}

\author{
By John Beek, Jr. and Arnold M. Sookne
}

\section{ABSTRACT}

The electrophoretic mobilities of four collagens and a gelatin were measured. One collagen was prepared from a limed steer hide; the other three were from the fresh hide, tendons, and bones of a steer. The differences between the mobilities are explained in terms of differences in the amidonitrogen contents of the various preparations. The isoelectric point of the unlimed-hide collagen is at about 7.0 on the $\mathrm{pH}$ scale.

\section{CONTENTS}

I. Introduction Page

II. Experimental materials and procedure

III. Results and discussion

IV. References

\section{INTRODUCTION}

Some properties of collagens prepared from the hide, tendons, and bones of a steer are being compared in connection with research work on leather at the National Bureau of Standards, with the object of determining to what extent the white fibrous connective tissues of these structures are similar. This paper reports measurements of the electrophoretic mobilities of particles of these collagen preparations, a gelatin made from the hide collagen, and a collagen sample prepared from a limed steer hide.

\section{EXPERIMENTAL MATERIALS AND PROCEDURE}

The preparation of the collagens has been described in earlier publications [1, 2]. ${ }^{1}$ A Wiley mill was used to grind the samples. The gelatin was made from the unlimed-hide collagen by heating in water for 18 hours at $90^{\circ} \mathrm{C}$.

In measuring the mobilities, the procedure described by Moyer [3] was followed in detail. Four types of buffer mixtures were used, namely, acetic acid with sodium hydroxide, and hydrochloric, phosphoric, and boric acids with potassium hydroxide. The ionic strength of all the buffers used was 0.02 molar. The acidities of the suspensions were measured with a glass electrode, the scale being fixed with reference to a value of 4.01 for the $\mathrm{pH}$ of a 0.05 molar solution of potassium acid phthalate.

The mobilities of gelatin were measured using suspensions of particles of Pyrex laboratory glass in solutions containing 0.01 percent of gelatin. Preliminary experiments at two acidities showed that, as the concentration of gelatin was increased beyond this value, there was no further change in the mobility of the particles.

\footnotetext{
1 Figures in brackets indicate the literature references at the end of this paper.
} 
At least 10 times of transit were measured for each suspension studied, the mean time being used for the calculation of the mobility. The measurements were made at $25^{\circ} \mathrm{C}$.

The amidonitrogen contents of the collagen preparations were compared by hydrolyzing samples in $0.1 \mathrm{~N}$ hydrochloric acid for 20 hours at $100^{\circ} \mathrm{C}$, neutralizing the solutions, and distilling the ammonia in the presence of an excess of magnesium oxide. As the amount of ammonia obtained varies with the conditions of hydrolysis and distillation [4], the results have no absolute significance. It is assumed here that the differences between results obtained under the same experimental conditions probably give a measure of the differences between the amounts of amidonitrogen in the several samples.

\section{RESULTS AND DISCUSSION}

The results of the determinations of mobility are shown in figure 1 . The relatively large vertical scale in this figure was used because of the small slopes of the curves for the collagen preparations.
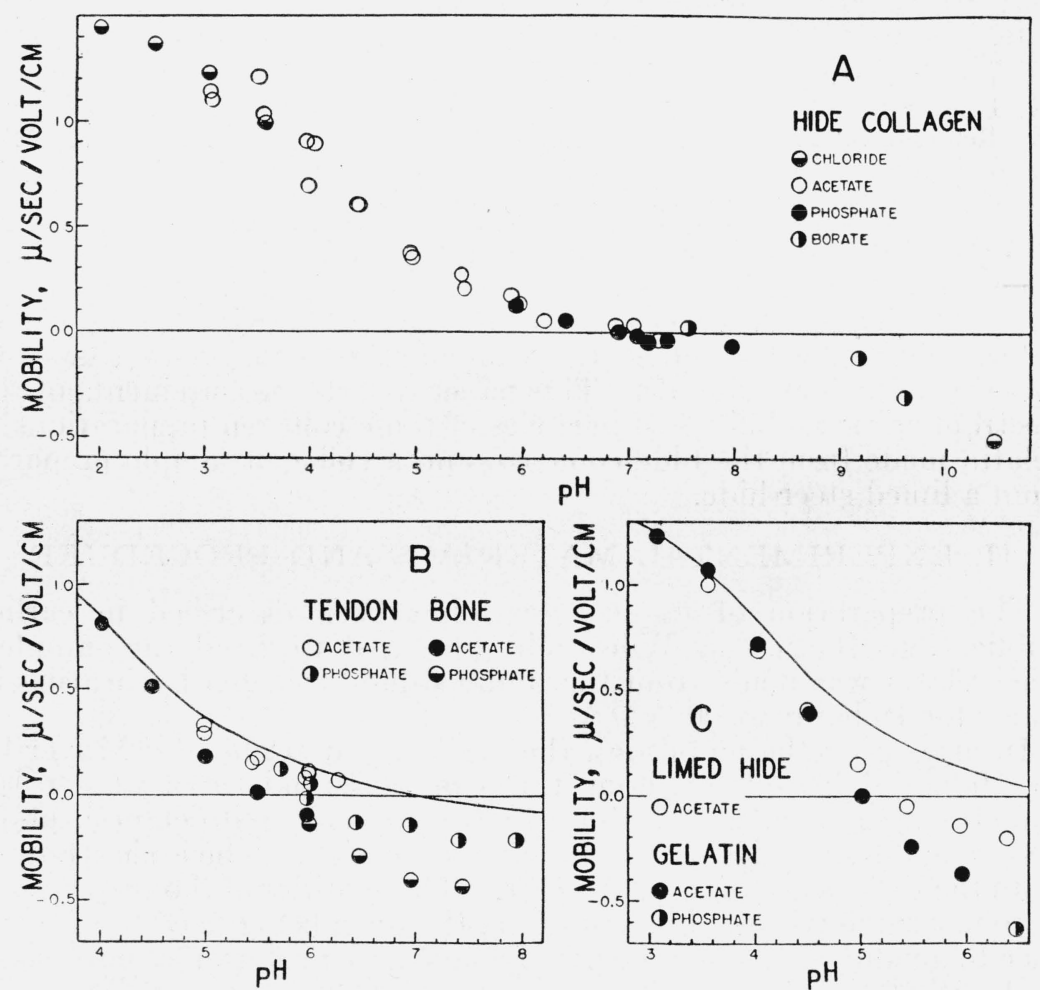

FIGURE 1.-The mobilities as a function of acidity.

Smooth curves are used in $B$ and $C$ to represent the mobility of the unlimed-hide collagen in order to avoid confusing these graphs with a large number of points.

The divergence of the mobilities at the lower acidities is qualitatively explained by the differences between the amidonitrogen contents of the various preparations, with the possible exception of the pair consisting of tendon and unlimed hide. The amidonitrogen 
contents of the tendon, bone, and limed-hide collagens are lower than that of the unlimed-hide collagen by $0.1,0.6$, and 0.6 percent of the total nitrogen, respectively. This decrease in the amidonitrogen content is accompanied by an increase in the number of free carboxyl groups, which in turn has the effect of increasing the net negative charge, if the acidity of the suspension is such that the free carboxyl groups are ionized to a considerable extent.

With regard to the isoelectric points, it may be pointed out that liming had the effect of shifting the isoelectric point of the hide collagen from near 7 to about 5.3 on the $\mathrm{pH}$ scale. The gelatin used in this work has a less acid isoelectric point, namely 5.0, than those for which measurements are reported in the literature $[5,6]$, presumably because no acid or alkali was used in its preparation from the collagen. Thus, it may be seen that the electrophoretically determined isoelectric point of a sample of collagen or gelatin depends on the processes used in its preparation.

In conclusion, three points should be emphasized. First, if it is assumed that all the collagens used were the same in their natural states, the relations among their mobilities are satisfactorily explained by the effect of the treatments used in "purifying" the samples. Secondly, the acid treatment used in decalcifying the bone collagen and the alkaline treatment used in depilating the limed hide produced materials which are closely similar with respect to their electrophoretic mobilities. Finally, the effect on the mobility produced in the formation of gelatin from hide collagen appears to be similar to the effect produced in the bone and limed-hide collagens by the acid and alkaline treatments.

\section{REFERENCES}

[1] J. Research NBS 21, 117 (1938) RP1119.

[2] J. Research NBS 14, 217 (1935) RP765.

[3] J. Bacteriology 31, 531 (1936).

[4] J. Biol. Chem. 112, 407 (1935).

[5] J. Chem. Soc. 192\%, 1250.

[6] J. Gen. Physiol. 15, 575 (1932).

Washington, June 6, 1939. 